\title{
Quelques aperçus sur l'électrification des chemins de fer en France
}

\author{
Par"I. BARBILIION, Directeur de l'Institul Polytechnique de Grenoble.
}

La France a toujours été un pays déficitaire en charbon. Les statistiques publiées en 1913, portant sur la consommation de charbon en 1912, statistiques intéressantes parce qu'elles correspondent à une période d'activité économique stable, accusaient une consommation de 60 millions de tomnes de charbon en France, contre une production de 40 millions. On évaluait à l'époque à 600 ou 800 millions de francs le côt du charbon acquis à l'étranger.

En plus des charges énormes représentées par le service des dettes dé toutes natures, par le budget spécial des dépenses recouvrables, notre budget actuel plie encore sous le fardeau des importations. L'importation du charbon coûte des milliards, l'importation des matières premières nécessaires à la fabrication du papier, de 800 à 900 millions, etc. Tout le problème du redressement consiste, en somme, à essayer de nous passer le plus vite et le plus complètement possible de ces importations qui effondrent notre change.

La consommation de charbon des chemins de fer français était de 9 millions de tonnes en 1912 . On évaluait à $5 \%$ l'augmentation annuelle du trafic. Par conséquent, en 1932, la consommation de charbon afférant aux chemins de fer aurait été de 18 millions de tonnes. Emus de cette progression croissante, et bien que d'importantes économies puissent être réalisées par ailleurs dans le chauffage domestique, dans les méthodes de la métallurgie industrielle, etc., par la substitution en grande quantilé de l'électricité au charbon, l'opinion publique, en même temps que le gouvernement, se préoccupaient, dès 1913, de cette question de l'électrification des voies ferrées. De nombreuses études, mises au point par des Commissions multiples, ont abouti au projet de la transformation, dans un délai d'environ vingt ans, du premier cinquième du réseau français, soit 8.200 kilomètres de voies ferrées, à peu près réparties par tiers entre le P.-L.-M., le P.-O. et le Midi. Cette tâche s'accomplira aussi rapidement que le permet la terrible crise apportée par la guerre. Une partie des lignes sont à électrifier, notamment Paris-Vierzon, du P.-O., parce qu'elles correspondent à une très grosse consommation de charbon, d'autres seront électrifiées parce que leur profil accidenté le nécessite, leur trafic étant d'autre part, suffisant pour justifier une transformation, telles seront les lignes Lyon-Grenoble, Culoz-Modane. C'est cette dernière qui est à électrifier la première sur le P.-.L.-M. Quant au Midi, dès 1921, il avait envisagé une électrification progresśive de son réseau, mais il a dû modilier son système technique. Ses premiers essais portaient sur un matériel monophasé. Or, aujourd'hui, les pouvoirs publics ont décidé l'adoption uniforme sur le réseau électrifié français du courant continu à 1.500 volts, bien que certaines sections, en réalité peu nombreuses, doivent marcher à 3.000 volts. Cette décision a été prise surtout après l'exemple fourni par l'Amérique.

Le problème était en effet délicat. Si la traction électrique des tramways, et même des métropolitains à faible rayon d'action, est à peu près définie comme type de matériel et comme caractéristiques de courant (courant continu à 500-750 volts pour les tramways, pouvant être porté à 1.200 volts pour certains métropolitains, par exemple le Nord-Sud, à Paris), la transformation des réseaux de chemins de fer suppose l'emploi de locomotives électriques nombreuses et puissantes, les puissances étant souvent de l'ordre de 3.000 chevaux. On voit que le problème de l'électrification des chemins de fer est toul à fait différent le celui relatif aux tramways et aux métropolitains. Il faut d'abord produire des stocks considérables d'énergie, le plus possible par voie hydraulique pour raison d'économie, en uliljsant les stations thermiques comme régulatrices ou comme appoint, transporter ces stocks d'énergic le plus loin possible à la plus haute tension possible, pour en perdre le moins pos. sible, et, enfin, distribuer aux locomotives l'énergic électrique nécessairc.

On doit donc partir de la locomotive, fixer la nature du courant qui Ini convient le mieux dans l'état actuel de la techniqup, puis remonter aux sources et fixer sous quelle forme ce courant peut être transporté et même produit. Or, pour des raisons techniques, que nous ne pouvons exposer ici, faute de place, on ne peut retenir que trois systèmes réellement pratiques, le triphasí, le monophasé et le continu.

Les deux premiers, bien qu'ils aient de nombreux partisans et aient fait l'objet d'importantes applications, ne semblent pas appelés à jouer le rôle capital entrevu lors des promières éludes relatives à l'électrification des chemins de fer. D'abord, ils in. fluencent d'une manière extrêmement fâcheuse les lignes à collrant faible voisines.

Certains réseaux suisses, espagnols, suédois, allemands et américains utilisent le monophasé, que les Chemins de fer du Midi ont cux-mêmes expérimenté, mais le système monophasé est lourd, peu puissant, les moteurs sont incomparablement moins robustes que ceux alimentés en triphasé ou même en continu. C'est une solution singuliere que toute l'habileté des Suisses semble à peine suffisante pour lui permettre d'effectuer un service normal sur les lignes du Loetschberg et du Gothard, cette dernière tout récemment électrifiéc.

Le triphasé est à la fois plus ancien comme application et plus généralisé que le monophasé. Il a été employé en grand pour la première fois sur les lignes de la Valteline, en 1901 (lignes équipérs par la Co Ganz, de Budapest), en même temps qu'une ligne concurrente, très comparable à celle de la Valteline de par son profil, sa longueur et son trafic, la ligne de Milan-Gallaratte, étail équipée par la Thomson en courant continu, après transformation dans des sous-stations, malheureusement très nombreuses, du courant alternatif triphasé émis par l'usine. A citer également la ligne de Berthoud-Thoune, en Suisse, installée également ell triphasé (1892), de mème qu'un certain nombre de chemins de fer de montagne, Zermatt-Gornergratt, Yungfrau, etc. Mais, c'est surtout la ligne du Simplon qui a servi d'école aux constructeurs, fournisseurs du réseau italien. Ce réseau est très important. Il comporte actuellement plus de 500 kilomètres électrifiés en triphasé (tout le réseau piémontais, les Giovi ct un certain nombre de sections en tunnel).

Le moteur triphasé est très robuste, mais peu maniable en ce qui concerne la vitesse de marche. Grâce à des arlifices asse\% délicats, on arrive à en réaliser deux, quelquefois quatre par des couplages différents de pôles, au rotor ou au stalor, mais cess solutions sont infiniment moins simples que celles utilisées en courant continu, où le grand nombre des moteurs permet des couplages série-parallèle particulièremont souples. Lo triphasé a été aussi utilisé en Amérique, mais faiblement. Beaucoup peñchent pour le courant continu.

Monophasé et triphasé ont encore un défaut commun. C'est 
de nécessiter pour Icurs moleurs des fréquences d'alimentation de 16 a 18 cycles par seconde, alors que l'accord s'est fait presque partout pour la fréquence 50 , en ce qui concerne la transmission de l'énergie. Il est bien évident qu'au fur et à mesure que le probleme de l'électrification prendra de l'importance, les usines affectées à la production des énergies de traction devront se souder aux autres usines, par l'intermédiaire de leurs lignes de transport et de postes de couplage. Or, ce couplage est impossible si l'on emploie des courants alternatifs de périodes différentes. Mais si l'on utilise du courant continu pour alimenter les locomotives, courant continu produit dans des sous-stations par la transformation du courant allernatif haule tension de
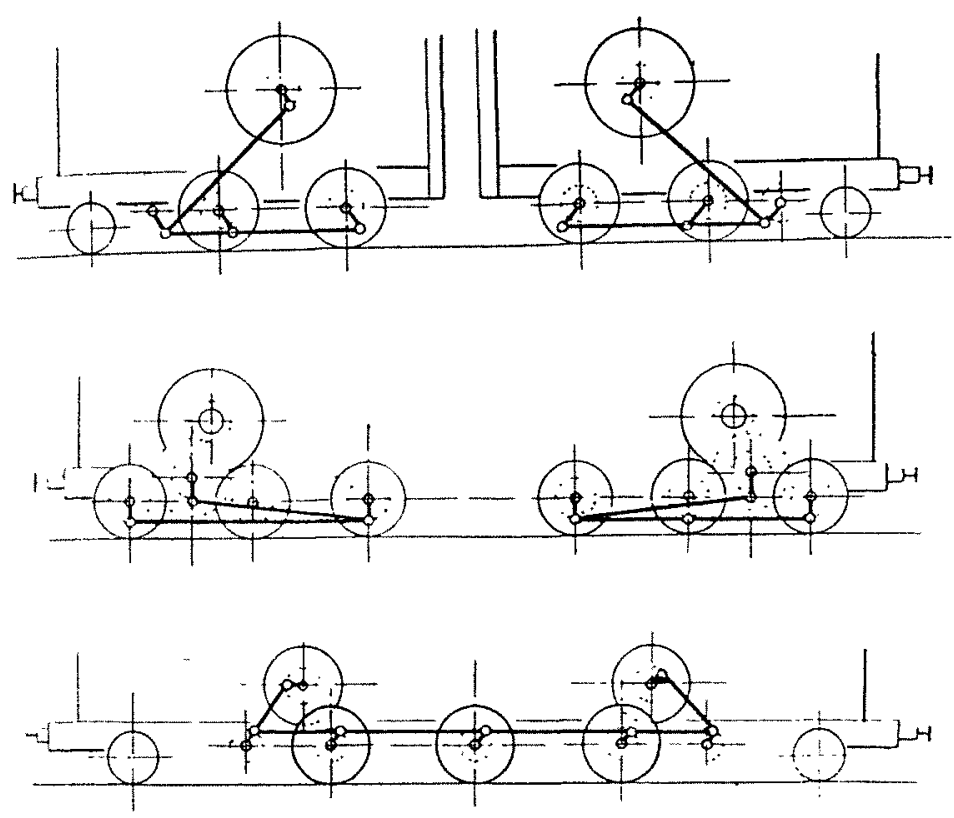

lig. 1. - Trucs de locomotives monophasées modernes.

1. Locomotives Lœtschberg A. E. G. et Oerlikon premier type. 3. Locomotive A. E. G. du Midi.

transport a 50 périodes, rien n'est plus simple que de coupler l'alternatif haute tension. Par contre, si l'on adopte la traction triphasée, comme le font nos amis italiens, e'est un supplice de Tantale que d'avoir des lignes de transport de traction à haute tension et à faible périodicité, côtoyant sans s'y relier les autres lignes de transport, toujours à haute tension, mais fonctionnant à la fréquence 50 . C'est ce qui explique les efforts faits depuis longtemps pour alimenter les locomotives triphasées avec du courant de périodicité 50 .

Qu'on nous permette, ici, d'ouvrir une parenthèse.

Cette insuffisance d'entente entre les divers réseaux, qui s'est traduite, notamment pendant la période de sécheresse extrême de l'année 1921, par un véritable désastre industriel, constitue dès lors, une source de gêne et de difficultés dont on conçoit l'importance. Dès lors, peut-on concevoir que, en dépit des efforts de lous les techniciens, une situation très grave, qu'un groupe d'ingénieurs-électriciens a signalée à plusieurs reprises à l'autorité militaire, existe dans la région parisienne du fait de l'adoption, depuis vingt ans, dans les secteurs, d'un mode d'utilisation de l'énergie tout à fait désuet, le diphasé ¿ 42 périodes, alors que dans tout le reste de la France (à l'exception près du triphasé à 25 périodes, qui dessert une partic du réscau de l'Energie du Littoral Méditerranéen), toutes les transmissions d'énergie fonctionnent en triphasé à 50 périodes?

Cdette situation anormale, pour ne pas dire plus, dans laquelle se débat la région parisienne, a failli provoquer, lors de la dernière guerre, un désastre national. Tout le matériel à commande électrique des usines de Paris, s’il avait dû être transporté effectivement en province, serait resté inutilisable !

On aurait pu supposer qu'un pareil danger, qui a fait frémir tous ceux, mèlés de près ou de loin aux organisations de la défense nationale, aurait fait l'objet, dès le lendemain de la guerre, d'études pressantes en vue d'y mettre fin. Rien n'en a été. C'est à peine, comme nous le disions tout à l'heure, si les milieux ofticiels et administratifs, sous la pression des techniciens. et aussi aujourd'hui, de l'autorité militaire, heureusement avertie, com. mencent à se préoccuper de la question.

Revenons aux applications du triphasé. Dès 1901-1902, sur la ligne militaire allemande de Berlin-Marienfeld-Zossen, sous l'impulsion de l'empereur Guillaume, dont les intentions déjà
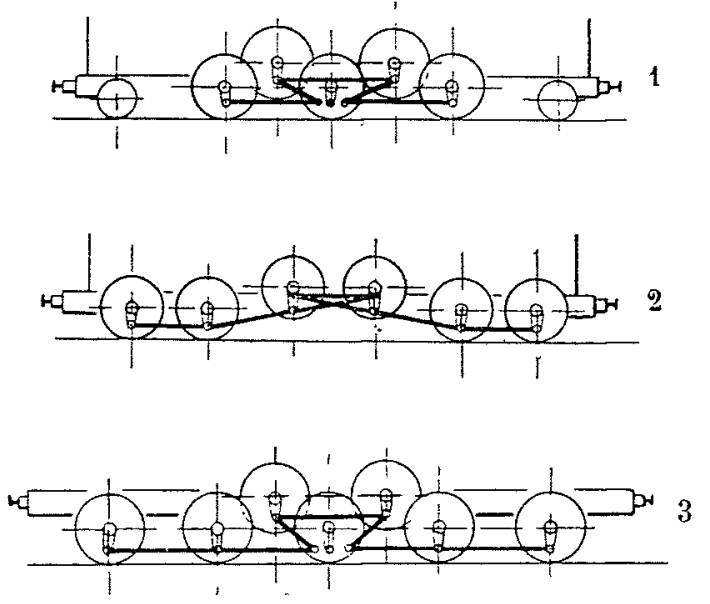

Fig. 2. - Trucs de locomotives triphasées modernes.

1 et 2. Simplon (Brown-Boveri). 3. Glovi (Westinghouse).

peu pacifiques à cette époque, s'accommodaient fort de tentatives destinées à intensifier les transports stratégiques, les deux grandes firmes allemandes Allgemeine-Elektrizitâts-Gesellschaft et Siemens-Halske établissaient des voitures automotrices et une locomotive d'essais destinées à fonctionner sous 10.000 volts triphasés à 50 périodes. Les difficultés rencontrées furent énormes. Néanmoins, le résultat fut atteint, puisque la locomotive Siemens, avec moteurs sans transformateurs, développa une vitesse de 217 kilomètres à l'heure sur une voie ferrée des plus médiocres.

Depuis ces expériences, à citer, les esprits se détachèrent de ce genre de préoccupation, mais tout récemment, les Italiens, sur un secteur de 40 kilomètres environ, entre Rome et Tivoli, ont exécuté des essais de traction triphasé à la tension de $\mathbf{1 0 . 0 0 0}$ volts et périodicité 50 . Il y a là une solution très intéressante, qui sera peut-être de nature, si l'expérience des faits la confirme, à diminuer quelque peu la portée des décisions de l'administration française, accordant la palme au courant continu.

La General Electric Company, dont on connaît les liens avec la Société Française pour le développement des procédés Thomson-Houston, après de nombreuses tentatives, a réussi entièrement l'électrification à 3.000 volts d'un premier tronçon allant de Harlowton à Avery (710 kilomètres), du Chicago-Milwaukee-Saint-Paul Railway, en Amérique, tronçon particulièrement dur, puisque sur une moitié à peu près de ce parcours, il correspond à la traversée des Montagnes Rocheuses. Ce tronçon appartient au grand réseau de 3.600 kilomètres qui s'étend de Chicago au Pacifique. Un deuxième tronçon vient d'être électri. 
fié, d'environ 310 kilomètres ; c'est le plus voisin du PacifiqueIl s'étend entre les villes de Seattle, Tacoma et Columbia Othello, mars sur ce tronçon, non seulement la General Electric, mais aussi la Suciété Westinghouse, ont été appelées en concurrence pour la fourniture du matériel locomoteur. C'est un matériel très spécial, très intéressant, d'une puissance considérable, puisque les locomotives électriques dépassent 3.000 chevaux el que les locomotives thermiques, que ces dernières étaient appelées à remplacer, développaient des puissances de plus de 3.600 .

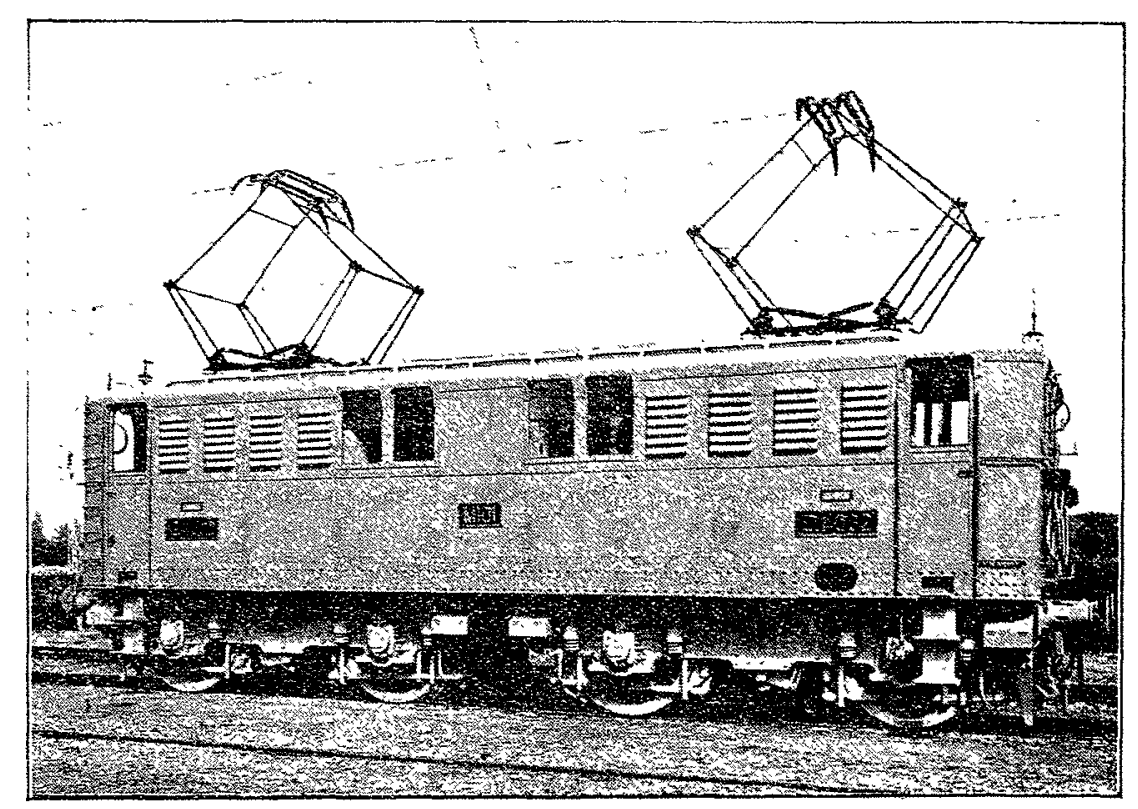

Fig. 3. - La première locomotive électrique française à courant continu des Chemins de fer du Midi. cè qui permet éventuellement de ne pas trop souffrir de chutes de tension ou meme d'accidents temporaires de lignes ou de sous. stations. On voit mal, plutôt on conçoit trop bien, ce qui arriverait en France en cas de telles perturbations, par exemple sur le secteur de Lyon-Arles, où le parcours des trains doit ètre réglé avec une précision quasi chronométrique. C'est sans doute ces considérations qui, sans qu'il y ait été fait allusion d'une manière explicite dans les rapports officiels, ont motivé l'adoption pres. que générale du 1.500 volts pour les réseaux français, avec quelques sections, peu nombreuses, équipées à 3.000 volts, mais, naturellement, possibilite de parcours et d'intercirculation de matériel des sections a 3.000 volts sur les sections à 1.500 .

En ce qui concerne plus particulièrement notre région du Sud-Est, la ligne de Culoz-Modane sert électrifiée, comme nous le disions tout a l'hour, dans un délai relativement bref.Elle servira, en particulier, de ligne d'expériences à la Compagnic P.-L..-MI, qui va y mettre en service un certain nombre de locomoteurs répondant naturellement dans leurs grandes lignes aux prescriptions d'unification des pouvoirs publics, mais dans lesquels des modifications de détail, destinées à permettre d'arriver an type standard, seront étudiées systématiquemenl. Il sera, en particulier, très curieux et très intéressint de comparer la nouvelle exploitation française it courant continu avec l'exploitation italienne voisint du Mont-Cenis, qui lui fait suite, à courant triphasí

II nous reste maintenant à examiner, pour teminer, deux points : d'abord, la question financière, au"moins dans ses grandes lignes, ef, ensuite, la répercussion que peut avoir l'électrification de nos chemins de fer sur la défenșe nationale.

Pour arriver à localiser de telles puissances motrices, il faut multiplier le nombre des essieux moteurs en les commandant chacun par un muteur dont l'induit est calé sur l'essieu, le système inducteur étant relié d'une manière plus ou moins élastique au truck. C'est là un caractère propre aux courants continus. La subdivision de la puissance entre de multiples moteurs présente de nombreux avantages. D'abord, chacun d'eux marche sous une puissance relativement faible, ce qui ménage le collecteur, puis l'installation sous le truck de moteurs de dimensions relativement réduites est aisée, enfin, la possibilité de couplage des divers moteurs, les uns avec les autres, entraîne la multiplication des régimes de vitesses économiques.

Le premier tronçon fut électrifié en 1915, le second en 1919 , le troisième, intermédiaire entre les deux premiers, est en voie d'exécution. Quand l'électrification sera complète, un réseau filiforme de 1.410 kilomètres environ, le plus grand réseau du monde, fonctionnera à courant continu 3.000 volts.

Examinons rapidement, en nous inspirant de cet exemple, si les conditions d'exploitation américaine sont suffisamment comparables à l'exploitation française, pour qu'on puisse transférer presque sans précaution le système d'électrification des Montagnes Rocheuses aux réseaux français.

Le grand réseau qui a servi de modèle aux études du Comité français ne laisse circuler que trois à quatre trains de marchandises par jour, très lourds du reste, plusieurs milliers de tonnes, et un très petit nombre également de trains de voyageurs de grande capacité. Le parcours est très long, l'horaire élastique,

En ce qui concerne le point de vue financier, signalons que les études d'électrification, d'aménagement des usines, d'achat do locomotives, etc., ont été faites en prenant les prix de 1913, et en les majorant dans le rapport 1,6. C'est évidemment un coelficient très faible par rapport au cont actuel des matériaux et de la construction électromécanique. Neanmoins, comme le programme doit se développer sur vingt ans, non à partir de 1912 , comme on l'escomptait au début, mais à partir de 1918 , il est permis d'espérer, sans trop y compter, que les prix s'abaissoront et que, si le coefficient de majoration est supérieur a $1, \hat{b}$ dans les premières annces, il lụi sera manifestement infériour dans les dernieres.

Les dépenses à engager, aux prix de 1912, sont rassemblées dans le tableau ci-apres : 


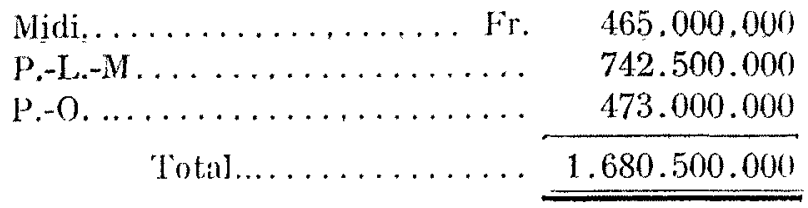

Ia Commission d'études ayanl cru devoir majorer ces chiffres de 1,6 pour tenir compte des suppléments de prix à prévoir, pour les travaux, pendanl la période de réalisation, les totaux ressortiront ainsi à :

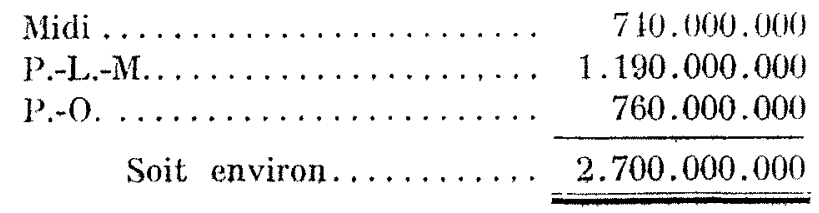

On remarquera, en consultanl le délail, qu'une grande partie de' cos dépenses n'est pas relative à l'électrification elle-même, par excmple celles correspondant à la construction des usines génératrices de certains réseaux, usines dont l'ampleur dépasse beaucoup les besoịns de la traction. De même, un certain nombre de travaux d'améliorations hydrauliques, d'irrigation, de (analisations électriques à haute tension, dintérêt général, qui figurent dans le total, n'intéressent que partiellement, ou même n'intéressent pas l'électrification proprement dite.

Si l'on tient compte du coefficient de majoration 1,6 , on trouve aiiisi un total de dépenses à investir égal à 2,7 milliards. Hâtonsnons dajouter que tous les techniciens estiment ces chiffres beaucoup trop faibles et le coût minimum de l'électrification drs 8.200 kilomètres intéressés au moins égal à 4 milliards.

du point de vue rémunération des capitaux, l'opération de l'électrification, sans être une opération brillante, se justifierait par suite des économies réalisées sur le personnel, le charbon, lentretien du matériel, les heures d'immobilisation, la réduction des ateliers et des dépòts, etc., tant que le coût du kilowattheure resterail inférieur à $\mathrm{fr}$. 0,04 , et que le coût de la tonne de (harbon resterait supérieur à 30 francs.

Si l'on adople, non pas 2,7 milliards, mais 4 milliards, les conchsions se modifient, mais pas autanl qu'on pourrait le croire, in raison de l'importance des dépenses mortes, en quelque sorte (dépenses indépendantes de la traction proprement dite), que l'on releve avec les machines à yapeur.

En loule conscience, on peut admettre yue, si l'état actuel de nos finances rend difficile la mise en jeu de capitaux imporlants, des milliards, pour l'aménagement du Rhône ou pour l'elcotrification des chemins de fer, au point de vile financier, la solution semble viable. C'est donc une question d'opportunité et de choix du moment pour la mobilisation des capitaux.

Terminons par un coup d'wil sur la répercussion que peut avoir l'électrificalion sur la défense nationale.

Dans une étude, fort documentée, parue dans le numéro lavili 1922 de la Revue Militaire Française, sous la signature de $M$. le colonel Le IIenaff, sur l'utilisation des chemins de fer français durant la dernière guerre, article auquel nous renverrons ceux que préoccupe cette si importante question des trans- ports par voie ferrée, envisagée sous une forme réellement rationnelle, l'auteur signale l'incoordination du début, les difficultés de toutes sortes qui ont présidé aux rapports des autorités militaires avec les directions de réseaux, elc. Il n'a pas fallu moins de loute la guerre pour remeltre un peu d'ordre et assurer Je plein rendement de cet organisme de nos voies ferrées, aujourd'hui bien au point. Si l'on fail abstraction, en effet, de la vingLaine de jours du début, qui correspondait à la mobilisation proprement dite, on constate avec le colonel Le I lénaff que presque rien n'avait été prévu pour la suite en matière de transports ; or, partant de cette considération capilale, que nous avons en mains un outil qu'on peut supposer aujourd'hui de premier ordre, semble-t-il désirable, au point de vue de notre sécurité, que l'on pousse l'électrification avec fièvre, au moins sur les parties de réseaux avoisinant nos fronlières ? On nous objectera que les Italiens, nos alliés, que les Suisses, neutres bienveillants, avaient déjà électrifié une partie de leurs chemins de fer avant la guerre, mais tout n'a pas été pour le mieux, qu'on le sache, en Italie et en Suisse, du fait de cette électrification, et lout aurait été, peut-être, beaucoup plus mal chez eux, si les Français avaient été leurs adversaires et avaient voulu détériorer leurs installations. Imaginons que sur les huit ou dix usines à peine desservant les 3 à 4.000 kilomètres élcctrifiés d'un réseau comme le P.-L.-M., un raid d'avions ou de dirigeables, bien conduits, vienne en annihiler la moitié. Ce n'est pas du tout improbable, c'est même extrêmement facile, affirmeront tous ceux qui, de près ou de loin, se sont occupés daviation pendant la guerre ou depuis la guerre. Voilà donc notre réseau électrifié immobilisé beauçup plus gravement que si quelques lignes de transmission d'énergie avaient été seules visées (les réparations de lignes s'effectuent bien vite, et, du reste, grâce aux interconnexions de réseaux, le dommage peut être très faible). Quant à la destruction des voies mécaniques, c'est un jeu pour des unités exercées de sapeurs de chemin de fer de les remettre en état en quelques heures ; le danger, c'est done la destruction d'un nombre rélativement important d'usines génératrices.

Le remède est simple : il consiste, en électrifiant avec prudence à conserver toujours un parc de locomotives thermiques, nombreuses, disponibles, en réserve à la première alerte, avec un personnel non moins entrainé de mécaniciens et de chauffeurs, de manière à pouvoir substituer au plus vite la traction à vapeur à la traction électrique sur les portions de réscaux que ne pourraient plus alimenter les usines génératrices.

Gagner du temps, tout est là. Peut-ètre, dans quelques années, les craintes de nouveaux conflits que nous pouvons avoir encore seront-elles tout à fail écartées. Jusque-là, c'est motre devoir de Français de ne pas vouloir sacrifier notre securité trop vite aux exigences du progrès scientifique.

Conservons à notre usage la maxime antique :

"Primum vivere, deinde philosophari".

Sachons, pour vivre, conserver la Force, dont l'exercice, suivant la sagesse grecque, n'est pas exclusif, de l'emploi de cette? arme intellectuelle qu'est la Science, et de cette autre arme morale, la Justice.

\section{NOTE DE LA RÉDACTION}

La Houille Blanche publiera, dans les prochains numéros, une élude sur le Barrage ilalien du Gleno, et la catastrophe que causa la rupture de ce barrage à la fin de l'ammée 1923.
Une première étude, purement descriptive, sera suivie, aussitôt paru le rapport officiel des experts, d'un article critique sur les causes de l'accident. 